\title{
Recent hot topics in contrast media
}

\author{
Henrik S. Thomsen
}

Received: 12 November 2010 / Accepted: 21 November 2010/Published online: 7 December 2010

(C) European Society of Radiology 2010

\begin{abstract}
This editorial reviews the way in which the facts related to the safety of iodinated and gadolinium based contrast agents have emerged over the last two decades. This is especially important given their ever increasing usage in modern computed tomographic (CT) and Magnetic resonance imaging (MRI) examinations. It also provides a very useful educational resume of this complex subject.
\end{abstract}

Keywords Contrast media - Adverse reactions .

Nephrogenic systemic fibrosis · Contrast induced nephropathy $\cdot$ Iodine based contrast media $\cdot$ Gadolinium based contrast media

\section{Introduction}

Since its start in 1991, European Radiology has published 65 papers in the category "contrast media". These include original papers, reviews and a few case reports. A total of 1,434 published papers contained the words 'contrast' and 'media'. The great majority of papers concern techniques for contrast medium administration, for example the timing of injection(s), injection rates and the choice of contrast

H. S. Thomsen

Department of Diagnostic Sciences, Faculty of Health Sciences, University of Copenhagen,

Blegdamsvej 3B,

DK-2200, Copenhagen N, Denmark

H. S. Thomsen $(\square)$

Department of Diagnostic Radiology 54E2,

Copenhagen University Hospital Herlev,

Herlev Ringvej 75,

DK-2730, Herlev, Denmark

e-mail: hentho01@heh.regionh.dk medium concentrations and doses. The remaining papers deal with safety issues, and this editorial focuses on these.

\section{Ionic high-osmolar versus non-ionic low-osmolar contrast media}

During the first years of European Radiology, discussions about whether the new non-ionic low osmolar iodine based contrast media were safer than the older high osmolar ionic contrast media ceased in Europe, because most radiologists had switched to the newer agents [1]. However, on the other side of the Atlantic, the discussions continued longer. The principal reason for this was that in the US the new agents cost 16 times more than the old ones, whereas in Europe the new agents were only four times more expensive. In the US, the prices of the old agents had decreased considerably in the 1980 s, but this did not happen in Europe. The few papers comparing ionic to non-ionic monomers indicate that this was no longer a hot topic in Europe.

\section{Contrast induced nephropathy (CIN))}

At the beginning of the second decade of European Radiology, the topic of contrast induced nephropathy (CIN) achieved new prominence because Tepel et al. [2] showed a marked protective effect of acetylcysteine against contrast induced nephropathy. The following year, however, the question was raised in European Radiology as to whether there was yet sufficient evidence for recommending the use of acetylcysteine [3]. The answer then was no, and it remains so. After nine years of multiple randomized trials, metaanalyses and analyses of the metaanalyses, Ellis and Cohan 
[4] concluded "There is little evidence to recommend the use of $\mathrm{N}$ acetylcysteine specifically to reduce the risk of CIN". A striking observation is that no one else has found such a large difference between the acetylcysteine arm and the control arm as Tepel et al. [2], even though Tepel only used a small dose of iodine based contrast medium $(75 \mathrm{ml})$, given intravenously for CT.

Three years after Tepel et al. [2] published their observation in the New England Journal of Medicine, the same journal published the finding of Aspelin et al. [5] that, in a small group of patients with moderate diabetic nephropathy undergoing angiography, the risk of CIN appeared to be less using the non-ionic dimer, iodixanol, rather than the non-ionic monomer iohexol. No subsequent studies have confirmed this finding and in a recent study involving the same type of patients, no difference was found between the non-ionic monomer and the non-ionic dimer [6]. The Aspelin study had a great impact on both research and academic debate. Despite the limited evidence, guidelines recommending the non-ionic dimer were even published [7]. About 14 randomized studies have been published, comparing the nephrotoxicity of one of the nonionic monomers with that of the non-ionic dimer given either intravenously or intraarterially, but no study comparing two non-ionic monomers has ever been published. Two metaanalyses $[8,9]$ have raised the possibility that the non-ionic monomer iohexol may be more nephrotoxic than the non-ionic dimer iodixanol, but no difference was found between the other monomers and the dimer. There is no pathophysiological explanation for these observations.

Much of the CIN data is based on intra-arterial administration of contrast agents. In view of the large numbers of enhanced CT examinations, it is important to note that the risk of significant CIN after intravenous nonionic contrast agents, such as iopamidol, iodixanol and iomeron, seems to be low even in patients with moderateto-severe renal insufficiency [10].

In the 1990s, it was widely believed that the gadolinium based contrast media used for Magnetic resonance imaging (MRI) were not nephrotoxic and they were used for conventional angiography and some CT studies to protect the poorly functioning kidney from the nephrotoxicity of iodine based contrast media $[11,12]$. However, the nephrotoxic potential of the gadolinium based agents is now recognized $[13,14]$. Experimental data in fact suggest that gadolinium based contrast media are more nephrotoxic than iodine based non-ionic monomeric contrast agents at equi-attenuating doses [14]. Despite the fact that the Gd atom attenuates $\mathrm{X}$-rays better than iodine at the most frequently used $\mathrm{kVs}$, the use of gadolinium based contrast media for radiography stopped completely after the link between gadolinium agents and development of nephrogenic systemic fibrosis was identified (see below).

\section{Off-label use}

When contrast agents are approved, the label describes the approved indications and particular circumstances of use, such as age, organ function or pregnancy [15]. The use of contrast agents outside the indications on the label is increasing rapidly. However, the terminology used in the product summaries is unclear and differs from product to product. The documents on which the labeled indications are based are confidential, often leaving the physician uncertain about whether he is using a contrast medium according to the labeled indication or off-label. For example, some vendors use the specific words 'MR angiography', while others use the indication "General MRI", making it unclear whether they are including vascular studies. This seems to be a specifically European issue, as the rules are different on the other side of the Atlantic. The precise role of Gd based agents for applications such as arthrography, sialography, etc. remains very unclear.

\section{Nephrogenic systemic fibrosis (NSF))}

Until the fall of 2006, most radiologists believed that all gadolinium based contrast media were of similar safety and were very safe. Nobody then knew about the disease nephrogenic systemic fibrosis (NSF), which was diagnosed in 1997 shortly after MR-angiography had been introduced as a safe and diagnostic examination. In 2006 and 2007, radiologists learned that NSF was a disabling disease occurring in patients with reduced renal failure after they had received the less stable gadolinium based contrast media. A review paper on this topic was published electronically in European Radiology only one month after submission and the printed version was published shortly after [16]. This was probably the fastest ever publication in European Radiology and the first warning about NSF in a radiology journal. A year later, it became clear that NSF could occur not only after gadodiamide but also after gadopentetate dimeglumine, but the prevalence seemed to be much lower [17]. According to PubMed, a total of 567 papers have been published with the key words "nephrogenic systemic fibrosis" up to November 1st 2010, nearly all since 2006. Hoppe et al. [11] stressed that conventional radiographic angiography with gadolinium based contrast media may play a role in the development of NSF in patients with renal insufficiency.

Since 2006, a number of experimental studies have been published [18]. Although we do not yet have a clear-cut understanding of the pathophysiology of NSF, there seems to be a consistent body of knowledge supporting the idea that the dissociation of low stability gadolinium-chelates, 
with the release of gadolinium, is an important factor in the pathogenesis of NSF. The non-ionic linear chelates are the least stable gadolinium based contrast media, and they can induce skin lesions and considerable retention of gadolinium in the tissues. By comparison, the very stable macrocyclic agents are associated with minimal retention of gadolinium in the tissues and no skin changes. Non-ionic linear chelates can stimulate the proliferation of human fibroblasts, increase the accumulation of collagen in the extracellular matrix, and stimulate the production of pro-inflammatory and pro-fibrotic cytokines and growth factors from monocytes. These effects seem to be gadolinium dependent, since gadolinium chloride can induce similar stimulant effects but the ligands of the gadolinium based contrast agents do not. The fibrogenic effects of the lanthanides were recognized as early as 1983 [19]. In-vitro studies have shown that lanthanides enhance the polymerization of skin collagen to a greater extent than calcium and may be involved in the promotion of fibril formation $[19,20]$. In the late 1980s, experimental studies in rats that had received multiple injections over three weeks showed skin lesions after gadodiamide but not after gadopentetate dimeglumine [21].

\section{Other effects of gadolinium}

The lanthanide series of elements, to which gadolinium belongs, do not occur naturally in the human body. Gadolinium has an ionic radius similar to that of the calcium ion. It is often used as an isomorphous replacement for calcium ions in biochemical studies. Gadolinium has the ability to mimic the effects of calcium ions, including activation of calcium dependent enzymes such as transglutaminase, an important enzyme in the development of fibrosis [18].

It has also been known for years that gadolinium can replace calcium in the hydroxyapatite within bone, which has a slow turn-over. Recently, there have been reports of patients developing NSF years after exposure to gadolinium based contrast media. Various diseases including uremia may affect bone turnover and then gadolinium may be released. There appears to be four times more gadolinium in the bones of patients with normal renal function after a non-ionic linear chelate than after a non-ionic macrocyclic chelate [22]. Retention of gadolinium in mouse tissue two weeks after injection was three times greater after a nonionic linear agent than after the ionic linear chelate [23]. Retention of gadolinium after injection of macrocyclic agents was minimal. Mice with renal impairment given radio-actively labeled Gd contrast agents had more radioactivity within their tissue after administration, and this increase varied according to which contrast agent was injected [24]. After seven days, mice with renal impairment that had received the ionic macrocyclic chelate had three times more radioactivity in their bone than control mice. However, mice with renal impairment that had received an ionic linear chelate or a non-ionic chelate had eight times and 24 times more radioactivity in their bone, respectively.

The accumulation of gadolinium in bone cannot be considered unimportant, because gadolinium is a heavy metal. This may well be the reason why the European Commission has decided that studies evaluating the potential for long-term accumulation of gadolinium in human bone should be undertaken [25]. Co-factors which may increase the risk of NSF such as calcium and phosphate levels at the time of administration of gadolinium based contrast media should also be studied and biomarkers evaluated. NSF may only be the tip of the gadolinium toxicity iceberg.

\section{Acute and late adverse reactions}

Contrast medium induced adverse reactions continue to be a problem [26]. They are unpredictable and diverse in nature. The terms 'acute' and 'late' reactions to contrast media refer to the time of onset of the reaction, but do not give any indication about the clinical features, the potential risk factors, or the methods of treatment and prevention.

Immediate reactions are the same for all types of contrast media (ultrasound agents, MR-agents and X-ray agents) and they have been recognized for many years $[27,28]$. The change from ionic high-osmolar to non-ionic iodine based contrast media reduced the incidence of reactions, with the result that there is now much less experience of treating moderate and severe acute non-renal reactions. Lightfoot et al. found that radiologists' knowledge of the use of epinephrine/adrenaline for the management of moderate and severe contrast medium adverse reactions is poor [29]. Therefore, regular training of staff and simple systems (crash cart in every room, clear instructions) are very important.

Late reactions develop after the patient has left the radiology department [30], and their relationship to the administration of contrast medium is therefore more difficult to recognize. Skin reactions are the most frequent late adverse effects of contrast media. They resemble skin reactions to other drugs and appear to be T-cell mediated. They are usually mild to moderate and self-limiting. Risk factors include a previous contrast medium reaction and Interleukin-2 treatment. Treatment is symptomatic and prophylaxis is generally not recommended [31].

\section{Conclusion}

During the last decade, interest in the safety aspects of contrast media has increased, largely because of contrast 
medium induced nephropathy and nephrogenic systemic fibrosis - both severe adverse reactions to contrast media in patients with reduced renal function. We now have some more insight into the pathophysiology of these adverse reactions, but there are still many unresolved issues. The incidence of nephrogenic systemic fibrosis has decreased dramatically since the most unstable agents have been avoided, and doses have been kept to a minimum. Contrast induced nephropathy remains a problem with no apparent difference in nephrotoxic potential between the available agents.

\section{References}

1. Thomsen HS, Archer JW, Schiermer L, Radensky PW (1998) The financial impact of Denmark's decision to restrict the use of highosmolar contrast media in intravascular radiographic procedures. Eur Radiol 8:321-322

2. Tepel M, van der Giet M, Schwartzfeld C et al (2000) Prevention of radiographic-contrast-agent-induced reductions in renal function by acetylcysteine. N Engl J Med 343:180-184

3. Thomsen HS (2001) Contrast-medium-induced nephrotoxicity: are all answers in for Acetylcysteine? Eur Radiol 11:2351-2353

4. Ellis JH, Cohan RH (2009) Reducing the risk of contrast induced nephropathy. A perspective on the controversies. AJR Am J Roentgenol 192:1544-1549

5. Aspelin P, Aubry P, Fransson SG et al (2003) Nephrotoxic effects in high-risk patients undergoing angiography. $\mathrm{N}$ Engl J Med 348:491-499

6. Laskey W, Aspelin P, Davidson C et al (2009) Nephrotoxicity of iodixanol versus iopamidol in patients with chroinic disease and diabetes undergoing coronary angiographic procedures. Am Heart J 158:822-828

7. Thomsen HS, Morcos SK (2006) Contrast-medium-induced nephropathy: is there a new consensus? A review of published guidelines. Eur Radiol 16:1835-1840

8. Heinrich MC, Häberle L, Müller V et al (2009) Nephrotoxicity of iso-osmolar iodixanol compared with nonionic low-osmolar contrast media: meta-analysis of randomized controlled trials. Radiology 250:68-86

9. Reed M, Meier P, Tamhane UU et al (2009) The relative renal safety of iodixanol compared with low-osmolar contrast media: a meta-analysis of randomized controlled trials. JACC Cardiovasc Interv 2:645-654

10. Thomsen HS, Morcos SK (2008) Risk of contrast mediuminduced nephropathy in high-risk patients undergoing MDCT - a pooled analysis of two randomized trials. Eur Radiol 18:891-897

11. Hoppe H, Spagnuolo S, Froehlich JM et al (2010) Retrospective analysis of patients for development of nephrogenic systemic fibrosis following conventional angiography using gadolinium-based contrast agents. Eur Radiol 20:595-603

12. Thomsen HS, Almén T, Morcos SK et al (2002) Gadoliniumcontaining contrast media for radiographic examination: a position paper. Eur Radiol 12:2600-2605

13. Thomsen HS (2004) Gadolinium-based contrast media may be nephrotoxic even at approved doses (case report). Eur Radiol 14:1654-1656
14. Elmståhl B, Nyman U, Leander P et al (2006) Gadolinium contrast media are more nephrotoxic than iodine media. The importance of osmolality in direct renal artery injections. Eur Radiol 16:2712-2720

15. Reimer P, Vosshenrich R (2008) Off-label use of contrast agents. Eur Radiol 18:1096-1101

16. Thomsen HS (2006) Nephrogenic systemic fibrosis: a serious late adverse reaction to gadodiamide. Eur Radiol 16:2619-2621

17. Leiner T, Herborn C, Goyen M (2007) Nephrogenic systemic fibrosis is not exclusively associated with gadodiamide. Euro Radiol 17:1921-1923

18. Morcos SK (2010) Experimental studies investigating the pathophysiology of nephrogenic systemic fibrosis; what did we learn so far? Eur Radiol. doi:10.1007/s00330-010-1951-z

19. Evans CH, Drouven BJ (1983) The promotion of collagen polymerization by lanthanide and calcium ions. Biochem $\mathrm{J}$ 213:751-758

20. Brouven BJ, Evans CK (1986) Collagen fibrillogenesis in the presence of lathanides. J Biol Chim 261:11792-11797

21. SMRM workshop on contrast enhanced magnetic resonance (1991) Discussion. Magn Reson Med 22:229-232

22. White GW, Gibby WA, Tweedle MF (2006) Comparison of Gd (DTPA-BMA) (Omniscan) versus Gd(HP-DO3A) (ProHance) relative to gadolinium retention in human bone tissue by inductively coupled plasma mass spectroscopy. Invest Radiol 41:272-8

23. Caravan P, Ellison JJ, McMurry TJ, Lauffer RB (1999) Gadolinium (III) chelates and MRI agents: Structure, dynamics and applications. Chem Rev 99:2293-2352

24. Wadas TJ, Sherman CD, Miner JH et al (2010) The biodistribution of $[153 \mathrm{Gd}] \mathrm{Gd}$-labeled magnetic resonance contrast agents in a transgenic mouse model of renal failure differs greatly from control mice. Magn Reson Med 64(5):1274-80. doi:10.1002/mrm.22553

25. COMMISSION DECISION of 1.7 .2010 concerning, in the framework of Article 31 of Directive 2001/83/EC of the European Parliament and of the Council, the marketing authorisations for Gadolinium-containing contrast agents for human use which contain one or more of the active substances "gadodiamide, gadopentetic acid, gadobenic acid, gadoxetic acid, gadoteridol, gadobutrol and gadoteric acid". http://ec.europa.eu/health/documents/ community-register/html/refh others.htm . Accessed November 1st 2010

26. Böhm I, Schild HH (2006) A practical guide to diagnose lesserknown immediate and delayed contrast media-induced adverse cutaneous reactions. Eur Radiol 16:1570-1579

27. Thomsen HS, Morcos SK (2004) Management of acute adverse reactions to contrast media. Eur Radiol 14:476-481

28. Morcos SK, Thomsen WJAW (2001) Prevention of generalized reactions to contrast media: a consensus report and guidelines. Eur Radiol 11:1720-1728

29. Lightfoot CB, Abraham RJ, Mammen T (2009) Survey of radiologist's knowledge regarding the management of severe contrast material-induced allergic reactions. Radiology 251:691696

30. Munechika H, Hiramatsu Y, Kudo S et al (2003) A prospective survey of delayed adverse reactions to iohexol in urography and computed tomography. Eur Radiol 13:185-194

31. Webb JAW, Stacul F, Thomsen HS, Morcos SK (2003) Late reactions to intravascular iodinated contrast media. Eur Radiol 13:181-184 